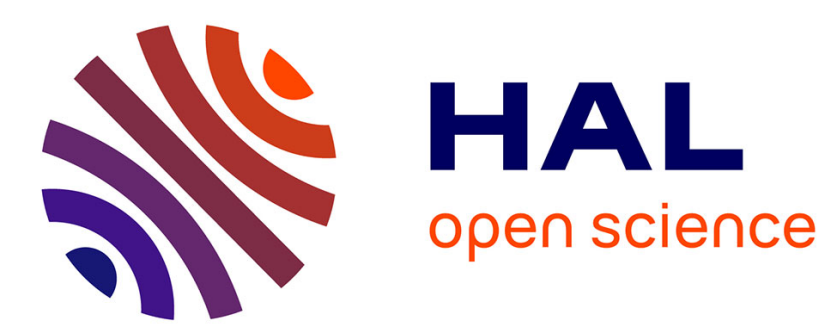

\title{
Géographie du développement sous le prisme de l'altermondialisme halieutique
}

Julien Noel

\section{To cite this version:}

Julien Noel. Géographie du développement sous le prisme de l'altermondialisme halieutique. C. Bouquet. Les géographes et le développement. Discours et actions, éditions MSHA, 2010. hal01654337

\section{HAL Id: hal-01654337 https://hal.science/hal-01654337}

Submitted on 4 Dec 2017

HAL is a multi-disciplinary open access archive for the deposit and dissemination of scientific research documents, whether they are published or not. The documents may come from teaching and research institutions in France or abroad, or from public or private research centers.
L'archive ouverte pluridisciplinaire HAL, est destinée au dépôt et à la diffusion de documents scientifiques de niveau recherche, publiés ou non, émanant des établissements d'enseignement et de recherche français ou étrangers, des laboratoires publics ou privés. 
Sous la direction de

Christian Bouquet

\section{Les géographes et le développement Discours et actions}

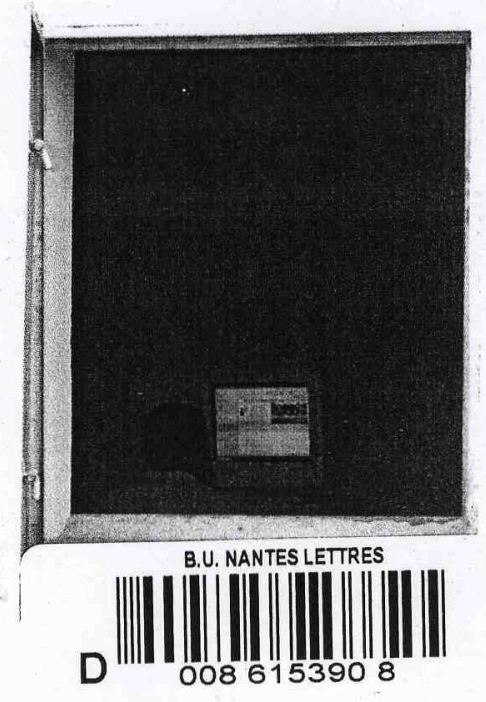




\title{
GÉOGRAPHIE DU DÉVELOPPEMENT SOUS LE PRISME DE L'ALTERMONDIALISME HALIEUTIQUE
}

\author{
Présentation
}

par Christian Bouquet

Julien Noël achève actuellement une thèse de géographie entamée en octobre 2004 à l'Université de Nantes au sein du laboratoire Géolittomer, LETG UMR 6554-CNRS. Son travail porte sur « L'altermondialisation et la dimension territoriale. Réflexions géographiques à propos des activités halieutiques et aquacoles \%。 L'objectif de cette recherche vise à montrer en quoi les mouvements contestataires de "pêcheurs à petite échelle " s'inscrivent dans une logique de réappropriation sociospatiale dans le processus de mondialisation. Ainsi porte-t-il un regard géographique sur l'altermondialisation, en privilégiant une approche géosystémique appliquée à deux espaces halieutiques et aquacoles (Bretagne Sud en France, Madagascar). Il insiste notamment sur la nécessité, pour cette coalition de mouvements sociaux, d'adopter un projet spatial davantage ancré localement afin de dépasser ses propres difficultés sociopolitiques et de contribuer à un développement différencié et solidaire des territoires.

Dans l'optique d'un développement davantage territorialisé de la part des militants altermondialistes du secteur de la pêche, il conclut la présente communication en insistant particulièrement sur certaines dimensions territoriales qui lui semblent révélatrices des enjeux à venir pour ces contestations. Aussi les discussions ont-elles porté sur la problématique des écolabels des produits de la mer comme perspectives de réappropriation (plus économique et stratégique que spatiale d'ailleurs). Au cours du débat, l'auteur a affirmé que certaines initiatives et outils de reconnaissance (IGP, $A O C)$ apparaissaient plus pertinents sur le plan géographique, notamment dans une démarche de territorialisation (terroir). 
L'accent a également été mis sur un autre élément intéressant : les stratégies de patrimonialisation qui gravitent autour de cette filière, tant du point de vue des ressources (biens publics, totémisation d'espèces, etc.) que des acteurs (festival de film sur la pêche, création de cité de la pêche, etc.).

À l'évidence, la contribution de Julien Noël s'inscrit au croisement du discours et de l'action... 


\title{
GÉOGRAPHIE DU DÉVELOPPEMENT SOUS LE PRISME DE L'ALTERMONDIALISME HALIEUTIQUE
}

\author{
Julien Noël
}

\section{INTRODUCTION}

« Un autre monde est possible ! » Véritable fer de lance dans l'affirmation des «nouveaux mouvements contestataires à l'heure de la mondialisation » (Sommier, 2001), ce slogan original intrigue tout autant qu'il interpelle. Pourtant, comme le souligne Eddy Fougier, chercheur à l'Institut français des relations internationales, si l'altermondialisation est « un thème de plus en plus connu du grand public, [...] les clichés et les caricatures continuent d'abonder à son propos » (Fougier, 2004). D'une manière générale, l'altermondialisation peut être présentée comme une « nouvelle mouvance sociale hétérogène contestataire de la globalisation néolibérale, à mi-chemin entre un néo-keynésianisme réformateur et un néo-marxisme plus radical » (Noël, 2004). Celle-ci élabore en effet toute une rhétorique et une dynamique protestataire prolifique en matière de développement, et qui ne reste pas sans conséquence sur ce " processus global de transformation sociale [censé conduire] à un accroissement des richesses associé à l'amélioration des conditions de vie » (Lévy et Lussault, 2003).

L'objectif de cette contribution consiste donc à s'interroger sur la conception géographique du développement proposée par cette mouvance contestataire, tout en basant notre réflexion sur l'une de ces multiples composantes, à savoir le mouvement altermondialiste halieutique, et notamment sur les organisations membres françaises et malgaches.

En effet, étant donné que le secteur d'activité halieutique demeure aujourd'hui fortement mondialisé, cela génère de multiples enjeux et 
diverses inégalités de développement, favorisant ainsi l'émergence d'une altermondialisation particulière, tant de point de vue de la sociologie de ses acteurs que de la sociographie de son discours et de ses actions. Néanmoins, nous verrons que cette dynamique contestataire semble souffrir de plusieurs déficits sur le plan spatial concourant ainsi à son relatif essoufflement, et auxquels les géographes semblent en mesure d'apporter des éléments de réponses, notamment en matière de territorialisation du discours et des actions.

\section{L'HALIEUTIQUE, TERRAIN DE PRÉDILECTION POUR L'ALTERMONDIALISATION}

Par définition, «l'halieutique » désigne tout ce qui a trait au champ de la pêche, c'est-à-dire cette activité de cueillette, de prélèvement ou de capture de ressources vivantes et sauvages (animaux et végétaux) aux dépens du milieu aquatique. De par les caractéristiques spécifiques de ces ressources, ce secteur d'activité présente de nombreux enjeux. Cependant, dans un contexte spatial de plus en plus mondialisé, ces enjeux font apparaître divers conflits et inégalités de développement.

L'HALIEUTIQUe, Un SECTEUR D'ACTIVITÉ AU CeEUR DE MULTIPLES ENJEUX DE DÉVELOPPEMENT

Comme le souligne très justement le géographe Jean-Pierre Corlay, la pêche comporte des enjeux considérables en matière de développement, et ce à divers plans - du secteur artisanal pour les pays du Nord et traditionnel pour les pays du Sud - et à diverses échelles - du local au global (Corlay, 2004).

Cette activité représente d'abord des enjeux sociaux en tant qu'activité créatrice d'emplois. En effet, selon les évaluations de l'organisation des Nations unies pour l'alimentation et l'agriculture (FAO), il y aurait dans le monde environ 15 millions de producteurs et 20 millions d'emplois induits, soit un total de 35 millions de fishworkers (travailleurs du poisson) travaillant au sein de la filière, de la capture à la commercialisation des produits. «En appliquant un coefficient multiplicateur de 4 à 5 par foyer, [le secteur halieutique] ferait vivre entre 140 et 185 millions de personnes » (Corlay, 2004). L'enjeu économique n'est pas moindre non plus quand on considère la valeur des exportations mondiales de produits de la pêche - autour d'une soixantaine de milliards de dollars US chaque année - comparée par exemple à celle de la viande (49 milliards). L'halieutique participe en outre à une certaine sécurité alimentaire : effectivement, le secteur représente environ $16 \%$ de la consommation mondiale en protéines animales et fournit, selon un rapport effectué en 1994 par l'ONG 
Greenpeace, près de $40 \%$ de la ration protéinique pour plus de $60 \%$ des populations des pays du Sud (Chaussade, 1997).

L'enjeu culturel est aussi présent car la pêche reste génératrice d'une multitude d'identités locales et régionales fortes ainsi que de savoir-faire artisanaux et traditionnels qui contribuent à une " socio-diversité qu'il est utile de préserver et qui donne lieu à des valorisations nouvelles comme le tourisme bleu » (Corlay, 2004). Enfin, l'enjeu spatial et territorial demeure d'une importance particulière puisqu'il permet une relative "stabilité sociospatiale, qui se mesure à l'aune des espaces construits et vécus par les communautés de pêcheurs à l'interface terre-mer et en mer » (Corlay, 2004).

Néanmoins, ces enjeux se répercutent différemment selon que l'on se trouve dans un pays développé ou dans un pays en développement. Par exemple, les pêcheurs artisans français sont déjà très largement engagés dans une économie marchande internationalisée tandis que la priorité des Malgaches reste la défense d'une pêche de subsistance. En outre, dans un contexte spatial de plus en plus globalisé, les nombreux enjeux géographiques véhiculés par ce secteur tendent à devenir de plus en plus conflictuels en termes de production, de partage et d'échange des richesses halieutiques et font apparaître de multiples inégalités de développement (Leroux et Noël, 2007).

L'HALIEUTIQUe, UN SECTEUR D'ACTIVITÉ DE PLUS EN PLUS MONDIALISÉ MAIS DE PLUS EN PLUS INÉGALITAIRE

Activité ancienne et ubiquiste, la pêche a connu tout au long du $\mathrm{xx}^{\mathrm{e}}$ siècle de profondes mutations techniques (motorisation, congélation) qui ont contribué à une « véritable explosion » du secteur dans un contexte de mondialisation grandissant. Le secteur halieutique participe donc pleinement à ce processus spatial, qui s'apparente selon les géographes à une intégration croissante et progressive d'espaces et d'hommes à l'échelle mondiale, et qui contribue pour certains d'entre eux à un double effet d'unification et d'uniformisation d'un monde façonné par un même mode de développement (Dolffus, 2001). Or, l'accélération et l'amplification actuelle de ce processus soumettent le secteur à des logiques spatiales instables et contradictoires. Désormais, cette activité libéralisée devient de plus en plus dominée par de puissantes multinationales, tant dans la production (Pescanova) que dans la distribution (Findus), concourant ainsi à une certaine déterritorialisation de l'ensemble de la filière (Noël, 2004).

Comme le rappelle Jean-Pierre Corlay, « si l'essor de la production halieutique demeure en grande partie imputable aux pays en développement, [celle-ci] ne leur profite pas totalement » (Corlay, 2004). En effet, si 
les captures des ressources marines demeurent encore très largement atomisées à l'échelle de la planète, la géographie de l'activité de pêche a connu de profonds bouleversements au cours des trente dernières années (Corlay et Chaussade, 2008). Si certains pays du Nord affichent toujours un niveau d'activité important (Canada, États-Unis, Europe, Japon, Russie), il semble bien que le centre de gravité de l'espace halieutique mondial ait très largement glissé vers les nouvelles puissances halieutiques d'Asie du Sud-Est (Chine, Inde, Indonésie,...) et d'Amérique latine (Pérou, Chili) qui regroupent près des deux tiers de la production (Corlay et Chaussade, 2008).

Néanmoins, la filière reste marquée par des phénomènes spatiaux de concentration que l'on retrouve aussi bien au niveau des zones de distribution que de consommation. «Jamais la mondialisation n'a été aussi forte que maintenant en matière d'échanges de produits de la mer » (Corlay et Chaussade, 2008), ces derniers figurant parmi les denrées les plus commercialisées à l'échelle du marché international (près de $40 \%$ de la production). Néanmoins, le négoce des produits halieutiques apparaît profondément déséquilibré spatialement et se traduit pour Jean Chaussade par « un véritable transfert de protéines » dans la distribution et la consommation de la rente aquatique au profit d'une « demande insatiable » des pays du Nord, qui concentrent quasiment $80 \%$ des importations et s'approvisionnent sur des espèces à haute valeur ajoutée (crevette, thon, saumon) (Chaussade, 2004).

D'autre part, le calcul de l'indicateur de « disponibilité alimentaire» élaboré par la FAO, qui mesure la quantité de produits de la mer ingérée par un habitant d'un pays chaque année, masque en fait d'énormes disparités dans le partage de la rente aquatique. En effet, si la moyenne mondiale avoisine les $16 \mathrm{~kg} / \mathrm{hab}$./an, les populations des pays industriels consomment en moyenne $28 \mathrm{~kg} / \mathrm{hab}$. contre à peine $14 \mathrm{~kg} / \mathrm{hab}$. pour les pays en développement. À titre d'exemple, au cours des quarante dernières années, les disponibilités alimentaires annuelles en produits de la mer sont passées de 18 à $29 \mathrm{~kg} / \mathrm{hab}$. en France alors qu'elles n'ont progressé que de 5 à 7,5 kg/habitant à Madagascar (Noël, 2004).

Cette « marchandisation » du monde halieutique tend donc à mettre en concurrence des systèmes territoriaux différents et spécialisés, débouchant ainsi pour Laurent Carroué sur « une explosion des inégalités »: crises environnementales (surexploitation et gaspillage de la ressource), instabilités culturelles (érosion du patrimoine maritime, rivalités identitaires), fractures sociales (entre les secteurs industriel et artisanal), etc. (Carroué, 2002). Ces conflits et ces inégalités favorisent donc l'émergence de mouvements contestataires comme celui de l'altermondialisation halieutique, qui peut être assimilé à une sorte de « fédération à mi-chemin entre 
une organisation de type communautaire et une organisation de type syndical » (Alliance pour un monde responsable, pluriel et solidaire, 2004).

\section{L'ALTERMONDIALISATION HALIEUTIQUE : ACTEURS, DISCOURS ET ACTIONS}

Afin de faciliter la compréhension de l'altermondialisation halieutique, il convient au préalable d'examiner les conditions d'émergence de ce mouvement contestataire ainsi que la composition de ses membres, avant d'expliciter le contenu de son discours et de ses actions en matière de développement.

Les acteurs de LA CONTESTATION : Chronologie ET SOCiOlogie DeS ALTERMONDIALISTES DU SECTEUR

Schématiquement, la spatialité de l'altermondialisation halieutique se traduit par une production complémentaire de hauts lieux symboliques, organisés de manière réticulaire, dont Rome et Delhi demeurent, par métonymie spatiale, les pôles d'impulsion les plus représentatifs.

Dès 1984, suite à l'adoption du nouveau Droit de la mer, la FAO organise à Rome (Italie) une conférence mondiale sur la pêche. Confrontée aux stratégies spéculatives du secteur ainsi qu'à une grave crise de surexploitation de leurs pêcheries côtières, une centaine de pêcheurs artisans et traditionnels de plusieurs pays - à majorité du Sud - se réunit parallèlement (Le Sann, 2001). Ces acteurs se rendent bien compte que si, dans un environnement mondialisé, les actions menées aux échelles locales et nationales pour défendre leurs intérêts et ceux de leurs communautés demeurent indispensables, celles-ci ont de plus en plus besoin d'être appuyées au niveau international (AMRPS, 2004). Afin de consolider leurs organisations et de structurer leurs réseaux, ces pêcheurs marginalisés posent donc les bases d'un mouvement international de solidarité pour la promotion de la pêche artisanale et créent en 1986 un réseau d'appui aux organisations professionnelles, l'International collective in support of fishworkers (ICSF).

Près de dix ans plus tard, dans le contexte de l'adoption par la FAO d'un Code de conduite pour une pêche responsable, plusieurs organisations de pêcheurs se retrouvent à nouveau au Canada en 1995 (AMRPS, 2004). Cette rencontre débouche deux ans plus tard à Delhi (Inde) sur la construction d'une structure originale, le World forum of fishworkers (WFF), composée de diverses organisations nationales de pêcheurs et de travailleurs de la pêche comme le souligne la figure 1.

Ce Forum mondial des pêcheurs et des travailleurs de la pêche semble plutôt constituer la branche « syndicale » de l'altermondialisation 
halieutique, son objectif principal étant principalement « de défendre les droits fondamentaux des communautés de pêcheurs, la justice sociale et leur culture » (WFF, 2000). L'autre composante altermondialiste est plutôt constituée d'ONG internationales d'appui, intervenant dans des champs thématiques spécifiques : ONG catholique de coopération au développement (Comité catholique contre la faim et pour le développement (CCFD)), ONG de solidarité internationale (Fondation Charles Léopold Mayer pour le progrès de l'homme (FPH)), etc. (figure 2). Néanmoins, l'organisme d'appui le plus emblématique de ce mouvement alternatif demeure l'ICSF, créé en Inde dès 1986, sorte de réseau d'appui et d'échange entre organisations professionnelles du Nord et du Sud afin de les aider à sortir de leur marginalisation (Le Sann, 2001).

Cette ramification des acteurs se retrouve par ailleurs à des niveaux d'échelles inférieurs, comme l'atteste l'exemple des organisations nationales de pêcheurs français et malgaches rassemblées respectivement autour de la Branche française du forum mondial (BFFM) et du Collectif des organisations maritimes malgaches (COMM). Ces deux structures, créées en 2000 , sont en effet soutenues par diverses ONG d'appui, telles que le CCFD, la FPH, Terre des Hommes, Peuples solidaires et le Centre de réflexion, d'information et de solidarité avec les peuples d'Afrique, d'Asie et d'Amérique latine pour la première, et par le CCFD, l'ICSF et l'Apostolat de la mer pour la seconde.

Ces affinités entre acteurs se reproduisent également sur le contenu de leurs discours et de leurs actions. En effet, l'ensemble de ces contestataires se positionnent communément sur la problématique du développement, s'employant ainsi à promouvoir un «nouvel ordre halieutique » (Corlay, 2004), au moyen d'un altermondialisme critique, entendu ici comme « un agrégat de propositions alternatives et d'actions militantes » (Noël, 2004).

\section{LE DISCOURS ET LES ACTIONS DE LA CONTESTATION : SOCIOGRAPHIE DE L'ALTERMONDIALISME}

À l'instar des études générales menées par certains sociologues et politologues sur la mouvance altermondialiste (Fougier, Agrikoliansky, Sommier, etc.), force est de constater que l'altermondialisme promu par les halieutes contestataires s'inscrit davantage dans une logique de « réappropriation sociospatiale du processus de mondialisation plutôt que dans une logique d'émancipation ou d'opposition à celui-ci » (Fougier, 2004). Autrement dit, ce positionnement critique génère une appréhension particulière de la question du développement. En effet, l'altermondialisme halieutique semble avant tout militer contre le mal-développement du secteur engendré par les politiques libérales : privatisation de l'accès aux 
ressources (quotas individuels transférables), pillages via les transferts technologiques de flottes (accords de pêche), gaspillages via des pratiques inadaptées (rejet de prises accessoires, pêches minotières) ou prohibées (pêche illégale sous pavillon de complaisance), etc. Selon ces acteurs, le secteur des pêches s'orienterait de plus en plus vers une logique de profit, privilégiant le développement d'activités productivistes et industrielles déterritorialisées au détriment des activités artisanales et traditionnelles davantage à finalité vivrière. À titre d'exemple, pour $1 \mathrm{~kg}$ de crevettes pêchées dans les mangroves des pays tropicaux, ce sont entre 8 et $10 \mathrm{~kg}$ de poissons divers qui sont aussi prélevés puis rejetés faute de débouchés commerciaux suffisants (Chaussade, 1997). Pour les altermondialistes, les produits halieutiques sont donc avant tout considérés comme des denrées marchandes, source de profits, au détriment de ressources qu'il importe de préserver afin de garantir des moyens d'existence aux populations de pêcheurs (AMRPS, 2004).

Or, comme l'exprime très clairement le chercheur-militant John Kurien, " il faudrait maintenant un changement radical des manières de penser et des politiques pour corriger la situation » (Kurien, 2000). Selon ce chercheur indien, il apparait nécessaire de « réorienter l'activité vers des modèles de développement durable, où l'on devrait trouver un plus grand degré de justice, de participation et d'autosuffisance » (Kurien, 2000). Le préambule de la charte de l'Assemblée constituante du WFF confirme l'orientation alternative souhaitée par ces acteurs « de s'unir pour faire respecter les droits fondamentaux, l'équité sociale et la culture des pêcheurs et travailleurs de la pêche, pour proclamer l'océan comme source de toute vie et pour s'engager à assurer la pérennité de la pêche et des ressources aquatiques pour les générations actuelles et à venir afin de protéger leurs moyens d'existence » (WFF, 2000). Ces derniers restent convaincus que la réponse à la crise actuelle du secteur passe par une revalorisation et un renforcement de la pêche artisanale et traditionnelle. C'est dans cette optique que Kurien propose tout un ensemble de mesures visant à promouvoir un « développement communautaire » du secteur (Kurien, 2000), mesures très largement réutilisées dans le discours contestataire comme l'illustre la figure 3 .

À ce titre, l'une des initiatives les plus originales réalisées par les altermondialistes réside dans l'instauration depuis 1997 d'une Journée mondiale des pêcheurs célébrée le 21 novembre de chaque année, date à laquelle fut intronisé le Forum. Concrètement, cette journée consiste en une programmation d'actions multiples et évolutives (manifestations contre les engins de pêche destructeurs, distributions gratuites de poissons, conservation des mangroves, etc.), permettant à la fois de mettre en 
valeur les liens de solidarité qui se sont noués entre fishworkers à travers le monde mais également de mettre en exergue les questions d'actualité essentielles pour l'avenir du secteur (WFF, 2000). D'autres actions moins symboliques consistent en l'organisation par l'ICSF de rencontres internationales (Fortaleza en 2006) et régionales (océan Indien en 2001) entre militants ou bien encore en la participation au nom du WFF à quelques grandes conférences internationales (Sommet de la Terre de Johannesburg en 2002) ou à des manifestations altermondialistes comme celle de Porto Alegre en 2005. D'autres démarches en matière de développement sont également entreprises aux échelles nationales et locales, comme l'attestent la vaste campagne d'alphabétisation destinée aux fishworkers traditionnels malgaches pour l'année 2008 ou bien encore celle sur la sélectivité menée par les pêcheurs artisans chalutiers de Bretagne-Sud en 2006-2007 sur la langoustine du golfe de Gascogne.

Si l'altermondialisation halieutique semble faire preuve d'un certain dynamisme en matière de développement, celle-ci laisse tout de même apparaître certains signes d'essoufflement auxquels les géographes semblent être en mesure d'apporter quelques éléments de réponses.

\section{REGARD GÉOGRAPHIQUE SUR LE DÉVELOPPEMENT ET L'ALTERMONDIALISATION HALIEUTIQUE}

Actuellement, la dynamique altermondialiste halieutique semble être confrontée à trois déficits majeurs qui paralysent sa rhétorique et ses initiatives en matière de développement. Face à ces difficultés, une lecture géographique axée sur une meilleure territorialisation de l'altermondialisme nous semble pertinente pour stimuler à nouveau le mouvement.

\section{UN ESSOUFFLEMENT DE LA CAUSE CONTESTATAIRE DÛ À TROIS DÉFICITS MAJEURS}

En nous inspirant des travaux sur la mouvance altermondialiste en général, menés entre autres par le politologue Eddy Fougier, l'altermondialisation halieutique semble souffrir de trois principaux déficits, à savoir ceux de globalité, de popularité et de crédibilité (Fougier, 2004).

Le premier déficit apparent de cette altermondialisation halieutique concerne celui de globalité, traduisant une spatialité discontinue. En effet, le mouvement demeure un phénomène relativement concentré sur le plan temporel, se manifestant de manière sporadique le temps de quelques " grandes messes » (Chavagneux, 2005). En outre, en dépit de l'élargissement souhaité dans le discours contestataire, ces mobilisations alternatives se sont majoritairement déroulées sur un nombre restreint d'espaces nationaux (Canada, États-Unis, France, Italie, Belgique), au détriment des 
autres espaces, connectés partiellement et plus tardivement à la dynamique altermondialiste. L'analyse géographique du mouvement amène donc à relativiser le caractère émancipateur transnational de ce dernier en matière de développement mais tend au contraire à souligner la résistance des espaces nationaux. Cette échelle permet en effet aux organisations de conserver leur autonomie et leurs pratiques spécifiques, tout en se dotant d'une dimension supranationale qui lie ces luttes entre elles et leur confère en même temps une légitimité supplémentaire (Agrikoliansky et al., 2005).

À ce déficit de globalité s'ajoute celui de popularité sociale. En effet, si l'altermondialisation halieutique demeure à l'origine une initiative du Sud, force est de constater que celle-ci n'est pas parvenue, au cours de son développement, à s'ancrer suffisamment sur les continents africains, asiatique et océanien, alors que l'Asie concentre plus des trois quarts des fishworkers à l'échelle mondiale. Ce déséquilibre se retrouve également au sein du comité de coordination du Forum puisqu'on ne compte qu'un seul élu africain sur douze délégués participants. Le WFF ne représenterait d'ailleurs qu'une infime partie des actifs du secteur halieutique puisque « $99 \%$ des pêcheurs et travailleurs de la pêche n'ont aucun contact avec cette organisation [...] L'urgence est donc de les rejoindre et de chercher à les organiser " estime Alain Le Sann (Le Sann, 2001). Enfin, une lecture plus attentive de la composition des participants à cette mobilisation révèle de profondes disparités, certaines catégories d'acteurs (femmes, mareyeurs, employés d'usines) apparaissant largement sous-représentées au profit des organisations de pêcheurs.

Enfin, le troisième déficit essentiel concerne le manque de crédibilité de l'altermondialisme. Les disparités qui subsistent entre secteurs artisan et traditionnel (techniques de pêche, représentativité du Forum, etc.) suscitent de multiples crispations entre membres. L'exemple du rapprochement entre les communautés de pêcheurs asiatiques et l'ONG environnementaliste Greenpeace - et très largement désavoué par les organisations européennes et nord-américaines - semble illustrer de manière probante les divergences en matière de luttes, d'actions et de stratégies d'alliances qui existent réellement entre communautés artisanales et traditionnelles. En effet, à une culture d'organisation assez radicale axée sur des logiques d'affrontement envers les organismes étatiques et internationaux pour les premières semble répliquer une politique plus réformiste pour les secondes, basée davantage sur des stratégies de négociation avec ces mêmes institutions (Le Sann, 2001 ; Fougier, 2004). De même, il semble que l'impact de l'altermondialisme soit pour l'instant assez limité, notamment dans la portée des grandes campagnes menées par ces sympathisants. Celles-ci apparaissent davantage efficientes lorsqu'elles se fondent sur de 
propositions propres au secteur et appuyées par des relais nationaux (lutte contre les engins de pêche destructeurs, zone de réserve) alors que sur des campagnes plus « politiques » (souveraineté alimentaire, lutte contre l'OMC), l'influence de ces contestataires reste plus réduite.

Ces diverses limites trouvent sans doute leur cristallisation lors de la scission du Forum pendant la rencontre de Loctudy (Bretagne-Sud) en 2000. Dès lors, la branche syndicale du mouvement se subdivise en deux entités distinctes :

- d'un côté le WFF, qui regroupe les organisations de pêcheurs artisans d'Europe et des deux Amériques ;

- de l'autre, le World forum of fisherpeople (Forum mondial des peuples pêcheurs) rassemblant les pêcheurs traditionnels d'Asie, d'Afrique et du Pacifique (Maoris) plus ceux d'Espagne et des Antilles.

Si plusieurs perspectives d'avenir ont été envisagées pour redynamiser cette contestation et tenter de dépasser ces difficultés (forums régionaux, rapprochement avec d'autres acteurs de la société civile, etc.), une perspective intéressante selon nous consisterait en une meilleure spatialisation du mouvement afin de pérenniser ses revendications pour un autre développement possible du secteur halieutique.

\section{GÉOGRAPHIE DE QUEL DÉVELOPPEMENT POUR L'ALTERMONDIALISATION HALIEUTIQUE ?}

L'absence de spatialisation du discours et des actions sur le développement du secteur par les altermondialistes halieutiques ne doit pas empêcher les géographes de porter un regard disciplinaire sur ce mouvement alternatif et sur ces perspectives d'avenir. D'un point de vue géographique, celui-ci implique de fait une maîtrise renouvelée de l'espace par les divers acteurs sociaux (États, société civile, entreprises), ce qui induit une transformation des rapports spatiotemporels (développement durable) ainsi qu'un changement des rapports sociospatiaux (entre pays du Nord et pays du Sud). Mais pour participer réellement et efficacement à la construction d'un autre pôle de développement, et tenter d'en réduire ou d'en corriger ses diverses inégalités, cette mobilisation contestataire se doit, à notre avis, de replacer l'espace - et notamment le territoire - au centre de ses préoccupations sur le développement, afin de redynamiser son discours et de pérenniser ses actions (Noël, 2004). Cette territorialisation de l'altermondialisme pourrait s'articuler autour de quelques notions-clés - celles de gouvernance et de patrimoine par exemple - synonymes d'une meilleure appropriation spatiale de la question du développement par ces militants halieutiques. 
En termes de gouvernance, autrement dit cet « ensemble de processus et d'institutions qui participent à la gestion politique " (Lévy et Lussault, 2003), les diverses stratégies de regroupement associatif opérées récemment par les femmes de pêcheurs - aussi bien en Bretagne-Sud (Femmes entre terre et mer) que sur la côte est malgache (Fivapamina) - constituent selon nous une perspective intéressante en matière de structuration des acteurs pour peser davantage dans le processus de décision et de gestion du secteur.

Diverses actions sont également menées en matière de valorisation patrimoniale, entendue ici comme «l'ensemble des attributs, des représentations et des pratiques décrétés d'importance collective et censés mériter d'être transmis du passé, pour trouver une valeur dans le présent » (Lévy et Lussault, 2003). Á ce titre, les démarches de labellisation et de certification entreprises ces dernières années par certains acteurs de la filière (bar de ligne breton, crevettes malgaches) laisse présager des perspectives de développement encourageantes. En outre, la mise en place du centre de découverte Haliotika par les pêcheurs artisans du Guilvinec (BretagneSud) afin de faire la promotion touristique d'une " cité de pêche » (visites guidées de la criée, dégustations culinaires, etc.) nous apparaît particulièrement probante dans le but de maintenir et de perpétuer l'identité maritime de l'activité.

\section{Conclusion}

Au terme de notre analyse, nous avons voulu montrer que l'activité de pêche présente de multiples enjeux et inégalités en matière de développement qui, dans un contexte fortement mondialisé, favorisent l'émergence d'une altermondialisation originale et critique, tant sur le plan de ses acteurs que du discours et des actions proposés. Toutefois, si cette contestation interpelle les géographes sur le manque conceptuel de spatialisation, elle ne doit pas empêcher ces mêmes géographes de s'investir et de proposer une réflexion disciplinaire de leur part qui pourrait permettre à ces militants de participer plus efficacement à la construction d'une autre logique de développement de leur secteur.

De notre point de vue, l'altermondialisation halieutique devra dans un avenir proche résoudre deux difficultés essentielles, à savoir son approfondissement (sur les plans idéologique et politique) et son élargissement (sur les plans social et spatial). Sans cet effort de structuration, le risque de récupération demeure réel. Assurément, le système halieutique actuel, fortement globalisé et libéralisé, montre déjà sa capacité de résilience, c'est-àdire sa capacité à intégrer les différentes formes de critiques sans changer foncièrement de structure qualitative, tout en se rendant écologiquement, 
socialement et culturellement plus acceptable (cf. le débat autour des écolabels). De fait, seule une culture de responsabilité mondiale qui combine prise de conscience de la dimension mondiale des problèmes et ancrage des solutions dans un territoire précis permettra de relever les défis de la mondialisation ainsi que de rétablir une certaine cohésion sociospatiale du secteur.

\section{Biblographie}

Agrikoliansky Éric, Fillieule Olivier, Mayer Nonna, 2005, L'Altermondialisme en France. La longue histoire d'une nouvelle cause, Paris, Flammarion, $371 \mathrm{p}$.

ALLIANCE POUR UN MONDE RESPONSAbLe, PLURIEL ET SOlidaIRE (AMRPS), 2004, La Pêche en eau trouble? Propositions pour une gestion durable des ressources marines, Paris, Éditions Charles Léopold Mayer, Cahiers de propositions pour le xxi ${ }^{e}$ siècle, $\mathrm{n}^{\circ}$ 9, $193 \mathrm{p}$.

CArroué Laurent, 2002, Géographie de la mondialisation, Paris, Armand Colin, 254 p. Chaussade Jean, 1997, Les Ressources de la mer, Paris, Flammarion, coll. Dominos, $127 \mathrm{p}$.

Chavagneux Christian (dir.), 2005, L'Altermondialisme a-t-il un avenir ? Les problèmes, les critiques, les pistes de renouveau, L'économie politique, Paris, Alternatives Économiques, $\mathrm{n}^{\circ} 25$, janvier, $112 \mathrm{p}$.

Corlay Jean-Pierre, Chaussade Jean, 2008, «Les échanges de produits de la mer : évolutions et enjeux des vingt dernières années », in GuIllaume Jacques (dir.), Les Transports maritimes dans la mondialisation, Paris, L'Harmattan, p. 159-184.

Corlay Jean-Pierre, 2004, « Du poisson pour se nourrir, du poisson pour vivre : les enjeux de la pêche et de l'aquaculture à l'aube du $3^{\circ}$ millénaire $», 15^{\mathrm{e}}$ Festival international de géographie, « Nourrir les hommes, nourrir le monde. Les géographes se mettent à table », Saint-Dié-des-Vosges, 30 septembre 2003-3 octobre 2004. En ligne sur le site du Festival international de géographie. Disponible sur http://fig-st-die.education.fr/actes/ actes_2004/corlay/article.htm

Dollfus Olivier, 2001, La Mondialisation, $2^{\mathrm{e}}$ édition, Paris, Presses de Sciences Po, coll. La bibliothèque du citoyen, $167 \mathrm{p}$.

Fougrer Eddy, 2004, Altermondialisme, le nouveau mouvement d'émancipation ?, Paris, Éditions Lignes de repères, $174 \mathrm{p}$.

Kurien John, 2000, « 14 mesures pour le développement durable et équitable de la pêche artisanale à petite échelle », Lorient, La Lettre d'information trimestrielle du groupe Pêche et Développement, CRISLA, $\mathrm{n}^{\circ}$ 52, $3^{\mathrm{e}}$ trimestre, p. 2-4.

LÉvy Jacques, Lussault Michel (dir.), 2003, Dictionnaire de la géographie et de l'espace et des sociétés, Paris, Belin, 1034 p.

LE Roux Sylvain, NoËL Julien, 2007, « Mondialisation et conflits autour des ressources halieutiques ", in Le Billon Philippe, Serfati Claude (dir.), Écologie \& Politique, Paris, Syllepse, $n^{\circ} 34$, p. 69-82.

LE SANN Alain, 2001, « De Rome à Loctudy : la naissance difficile d'un mouvement international des pêcheurs artisans $»$, Nantes, Cahiers Nantais, IGARUN, $\mathrm{n}^{\circ} 55-56$, janvierjuillet, p. 281-288. 
NoËL Julien, 2004, Les Enjeux géographiques de l'altermondialisation dans la production et les échanges des produits halieutiques et aquacoles. Réflexions méthodologiques bibliographiques, Nantes, Université de Nantes, mémoire de DEA en géographie, 103 p.

Sommier Isabelle, 2001, Les Nouveaux Mouvements contestataires à l'heure de la mondialisation, Paris, Flammarion, coll. Dominos, $128 \mathrm{p}$.

WFF, 2000, Assemblée constituante du WFF (Loctudy, France, 2-6 octobre 2006). Actes. Annexe 1 : la genèse du mouvement, Lorient, document interne au WFF, CRISLA, $108 \mathrm{p}$. 


\section{AnNeXes}

\section{Carte 1 - Le World forum of fishworkers :} rencontres et organisations membres

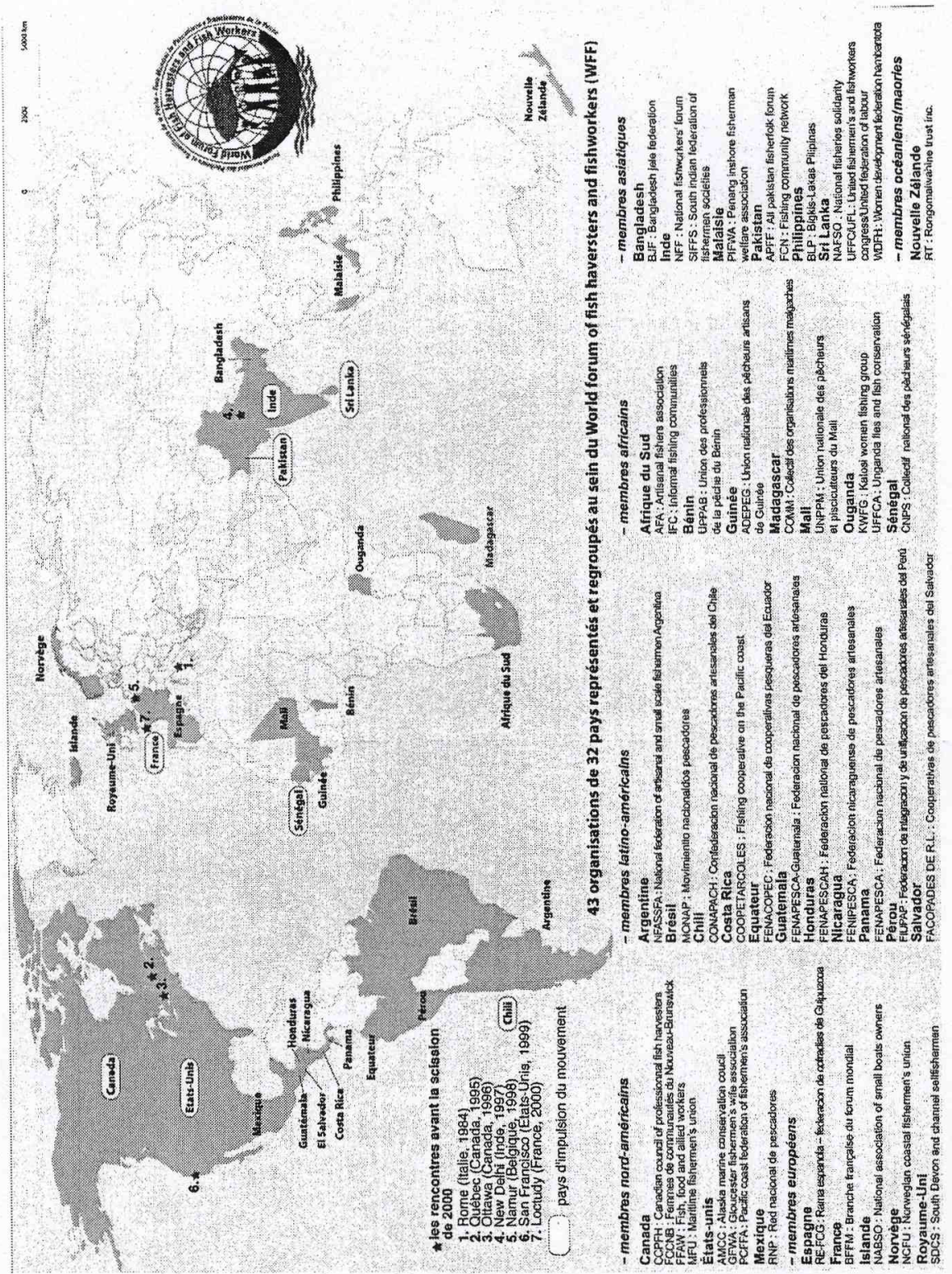

Source: Julien Noël, IGARUN, Geolittomer, 2006 d'après Le Sann, 1995, site internet de Pêche et Développement. 
Figure 1 - Principaux acteurs de l'altermondialisation halieutique
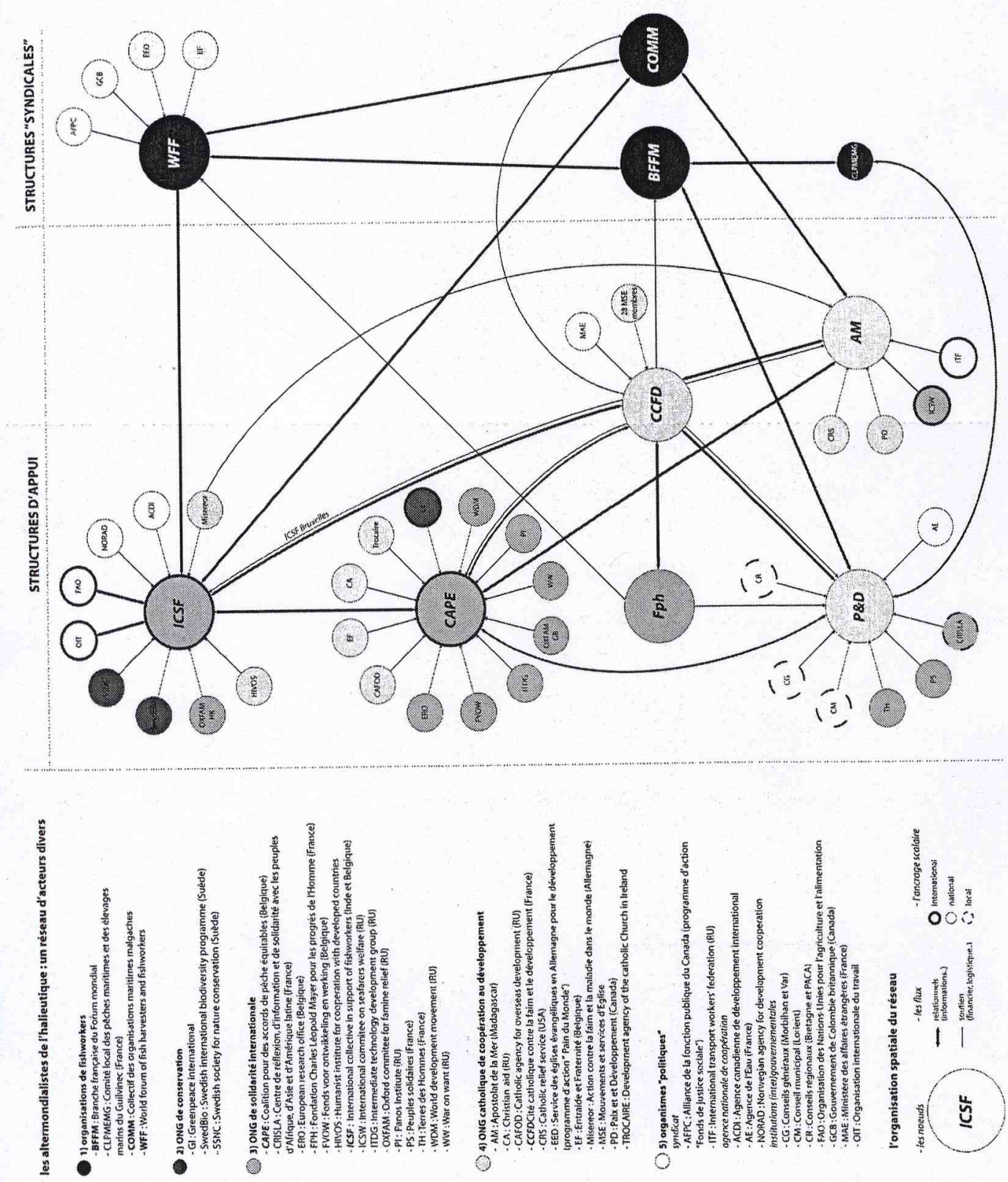

Source: Julien Noël, Geolittomer, LTG UMR-6554-CNRS d'après WFF, 1998 ; 200a ; entretiens personnels. 
Figure 3 - Le nouvel « ordre halieutique » des altermondialistes

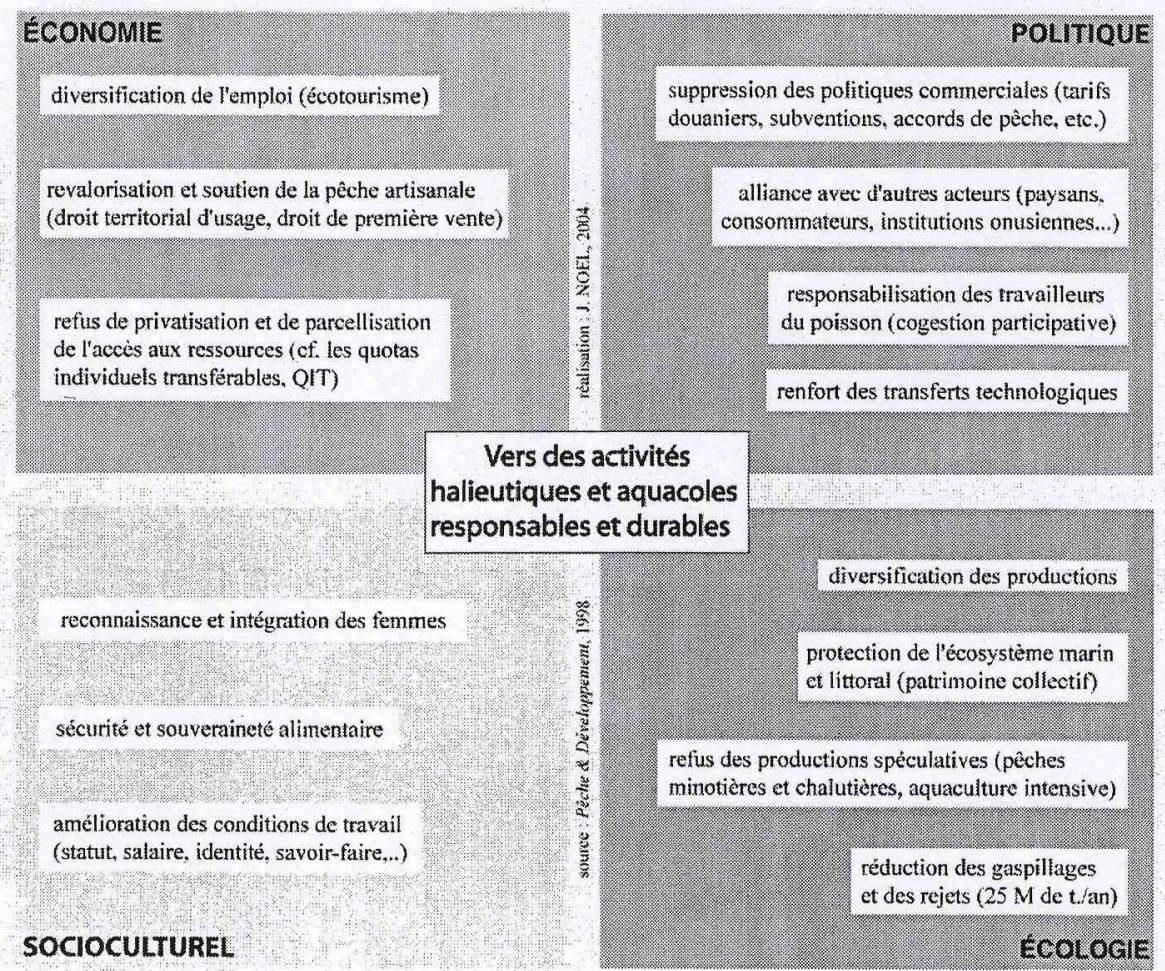

